\title{
Enhancement and decrease of critical current due to suppression of superconductivity by a magnetic field
}

\author{
D. Y. Vodolazov, $, 2, *$ D. S. Golubović,,$^{3,}$ F. M. Peeters, $, 2,+$ and V. V. Moshchalkov ${ }^{3}$ \\ ${ }^{1}$ Institute for Physics of Microstructures, Russian Academy of Sciences, 603950, Nizhny Novgorod GSP-105, Russia \\ ${ }^{2}$ Departement Fysica, Universiteit Antwerpen (CGB), Groenenborgerlaan 171, B-2020 Antwerpen, Belgium \\ ${ }^{3}$ Nanoscale Superconductivity and Magnetism Group, Institute for Nanoscale Physics and Chemistry (INPAC) K.U. Leuven, \\ Celestijnenlaan 200D, B-3001 Leuven, Belgium
}

(Received 20 April 2007; revised manuscript received 20 June 2007; published 5 October 2007)

\begin{abstract}
We present experimental results on the transport properties of a superconducting aluminum loop connected to reservoirs. As function of the applied magnetic field, an unexpected behavior is found - a steep enhancement (for temperatures close to the critical temperature) and a sharp drop (at lower temperatures) of the critical current at some value of the magnetic field $B_{a}^{*}(T)$. These effects are a consequence of a sudden suppression of superconductivity at $B_{a}=B_{a}^{*}$ in the banks to which the loop is attached by the current leads. As a result, the normal metal-superconductor boundaries appear at the ends of the current leads and the quasiparticle distribution function $f(E)$ in the superconductor deviates strongly from its equilibrium value. Calculations based on the Usadel equations show that the critical current should be enhanced at high temperature (due to the penetration of the normal current from the normal metal-superconductor boundaries), whereas at low temperatures, the suppression of the order parameter by the nonequilibrium $f(E)$ dominates and the critical current decreases. The latter provides a direct experimental verification of the recently proposed mechanism of the destruction of superconductivity by an applied voltage [R. S. Keizer, M. G. Flokstra, J. Aarts, and T. M. Klapwijk, Phys. Rev. Lett. 96, 147002 (2006)].
\end{abstract}

DOI: 10.1103/PhysRevB.76.134505

PACS number(s): 74.78.Na, 73.23.-b, 74.20.De, 74.25.Op

\section{INTRODUCTION}

During the past decade, several works have been published where it was shown that by applying a magnetic field to the superconductor, one may restore or even induce superconductivity. ${ }^{1-4}$ This effect is mainly connected with the compensation of the intrinsic magnetic field in the material. For example, in Ref. 1, the magnetic field of the magnetic dot is compensated by the external magnetic field, while in Refs. 2 and 3, the external magnetic field orders the magnetic impurities in the superconductor, which decreases their depairing effect on superconductivity. In Ref. 4, the external magnetic field compensates the effective exchange field of the organic conductor and it leads to the nucleation of superconductivity in the material.

In the present work, we study a physical system (a superconducting loop connected by current leads with bulk superconducting banks) where, even in the absence of an intrinsic magnetic field, the external magnetic field may enhance the superconducting properties of the system, in particular, its critical current. Previously, it was shown that the magnetic field can affect the superconducting properties via orbital and Zeeman (paramagnetic) effects. Here, we present a different mechanism where the conversion of part of the system (i.e., superconducting banks) influences the critical current in another part of the system (i.e., superconducting loop). In the temperature interval close to the critical temperature, it leads to a higher value of the critical current as compared to a loop coupled to superconducting banks, whereas at lower temperatures, it leads to an abrupt suppression of the critical current when the banks become normal. The obtained results can be explained by the nonequilibrium effects induced by the voltage drop between the normal banks.
The paper is organized as follows. In Sec. II, we present our experimental results. Sec. III gives our interpretation of the experiment, which we support by theoretical calculations that are based on a self-consistent solution of the Usadel equations. Finally, in Sec. IV, we present a discussion of the results, and in Sec. V, we make our final conclusions.

\section{EXPERIMENT}

Figure 1 shows a scanning electron microscope image of our experimental system: a $45 \mathrm{~nm}$ thick $\mathrm{Al}$ superconducting loop with current and voltage leads, used to investigate the transport properties. The inner radius of the loop is $728 \mathrm{~nm}$, outer radius equals $958 \mathrm{~nm}$, and left (right) voltage contact is $300 \mathrm{~nm}(250 \mathrm{~nm})$ from the loop. The length of the current

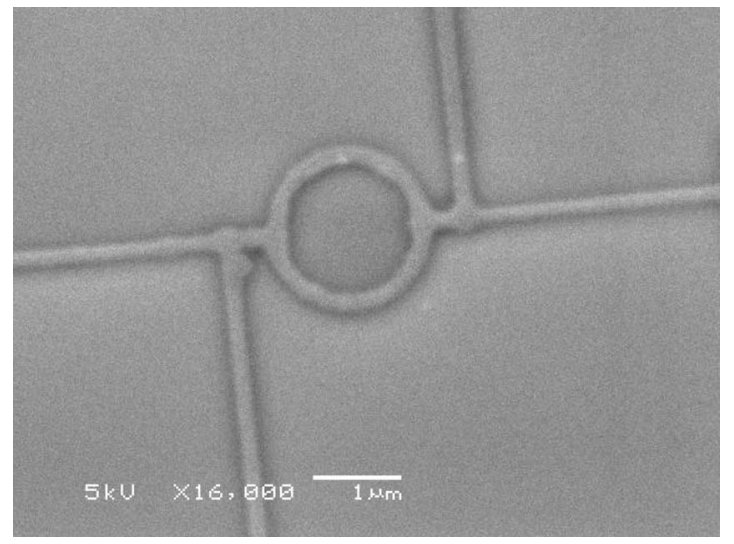

FIG. 1. A scanning electron microscope image of the aluminum loop with attached current and voltage electrodes. 


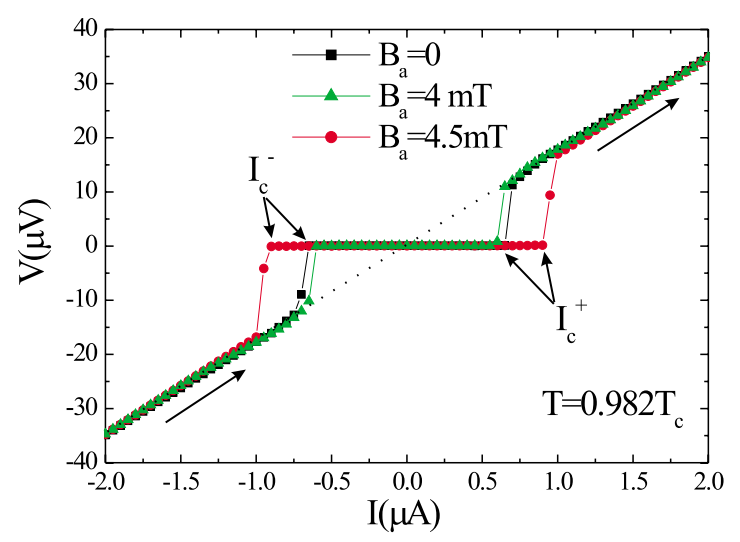

FIG. 2. (Color online) Current-voltage characteristics of the superconducting loop at different values of the magnetic field and $T$ $=0.982 T_{c}$. The dotted curve corresponds to the normal state of our system. At current $I_{c}^{-}$, the loop transits from the normal to the superconducting state, and at $I=I_{c}^{+}$, from the superconducting to the normal state.

leads, with width $230 \mathrm{~nm}$ (the same as the width of the loop), is about $5 \mu \mathrm{m}$, and beyond that point, they become wider and extend to $15 \mu \mathrm{m}$ before they touch the bulk contacts (not shown in the image). The transport measurements we performed in dc mode by sweeping a current from high negative values, sufficient to drive the system to the normal state, up to high positive values at a fixed value of the magnetic field and temperature. Figure 2 shows the current-voltage $(I-V)$ curves of the loop measured at $0.982 T_{c}$ at different applied magnetic fields. The dotted line shows the normal state $I-V$ curve. $I_{c}^{-}$stands for the critical current at the normal statesuperconducting transition, whereas $I_{c}^{+}$denotes the critical current of the superconducting-normal state transition.

Our experimental results are summarized in Figs. 3(a) and 3(b), where we show contour plots of the measured voltage as a function of the applied magnetic field and current for two temperatures, $T=0.982 T_{c}$ and $T=0.962 T_{c}$, respectively ( $T_{c}$ stands for the zero-field critical temperature). The central region (green) corresponds to the area where the voltage is practically equal to zero (purely superconducting state). For low magnetic fields, both critical currents $I_{c}^{+}$and $I_{c}^{-}$practically do not oscillate with applied magnetic field at both temperatures shown. A sharp transition to the normal state at $I=I_{c}^{+}$is observed and, equivalently, from the normal to the superconducting state at $I=I_{c}^{-}$. At temperatures very close to $T_{c}$ (i.e., $T>0.995 T_{c}$ ), oscillations of $I_{c}^{ \pm}$as function of $B_{a}$ are observed, as were found earlier in Ref. 5. In the region close to $T_{c}$, a smooth transition from superconducting to the superconducting resistive and, finally, to the normal state, and vice versa, were observed for $I>I_{c}^{+}\left(I_{c}^{-}\right)$, similar to the findings of Ref. 5.

At some magnetic field $B_{a}^{*}$ (see Fig. 3), there is a sudden change in the behavior of $I_{c}\left(B_{a}\right)$. Both positive $I_{c}^{+}$and negative $I_{c}^{-}$critical currents become larger than $I_{c}^{ \pm}(0)$ for temperatures close to $T_{c}$. On the other hand, the current $I_{c}^{+}$abruptly drops and $I_{c}^{-}$increases at $B_{a}=B_{a}^{*}$ for lower temperatures. At the same time, oscillations in the critical current appearsfor high temperatures in both $I_{c}^{+}$and $I_{c}^{-}$, and at low tempera-


FIG. 3. (Color online) Contour plot of the voltage drop $V$ at the loop as a function of the applied dc $I_{a}$ and the magnetic field $B_{a}$ measured at (a) $T=0.982 T_{c}$ and (b) $T=0.962 T_{c}$.

tures only for negative current, when a transition from the normal to the superconducting state occurs.

\section{THEORY}

The width of the current and voltage leads in our sample is much smaller than the coherence length $(\sim 117 \mathrm{~nm}$ at zero temperature) in the studied temperature range. It means that the critical magnetic field of the leads $B_{c} \sim \Phi_{0} / w \xi(T)$ is much larger than the critical field of the bulk superconductors $B_{c} \sim \Phi_{0} / \xi(T)^{2}$. A change in the behavior of our system occurs when the superconducting banks are driven to the normal state by the external magnetic field (at $B=B_{a}^{*}$ in Fig. 3 ). Actually, it is the only transition expected in our system at the given values of the magnetic field. In order to verify that this effect is indeed responsible for the experimental observations, we should compare the properties of our system in two limits: when the banks are in the superconducting and normal states.

Let us first consider the case that the banks are in the superconducting state, which, according to our assumption, occurs for magnetic fields $B<B_{a}^{*}$ (see Fig. 3). When we apply a current to our system, the maximal current density is reached in the current leads. In the loop area, the current density is half of the one in the superconducting leads because of the two different paths that the current can follow. In the presence of an applied magnetic field, an additional screening current appears in the loop and adds to the transport current in one arm of the loop and subtracts from the transport current in the other arm of the loop. Because we did not observe any oscillations of the critical current as 


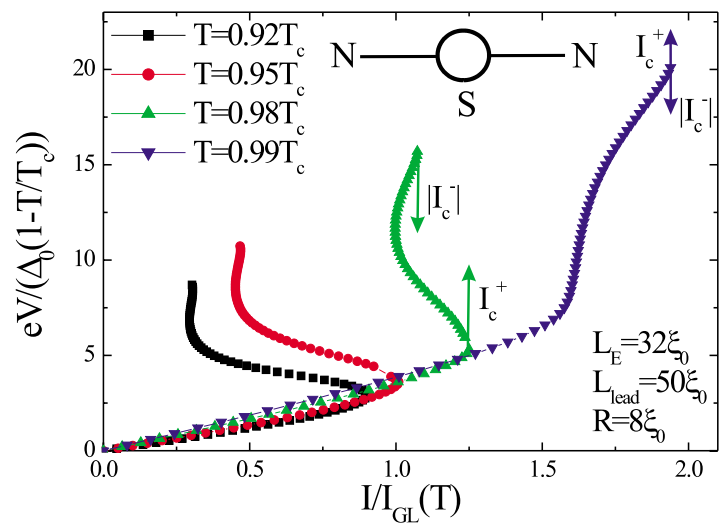

FIG. 4. (Color online) Current-voltage characteristics of our model system at different temperatures, obtained in the voltagedriven regime $\left(B_{a}=0\right)$. The curves stop at the points where our model system transits to the normal state. Arrows on two of the curves indicate the values of the negative $\left|I_{c}^{-}\right|$and positive $I_{c}^{+}$critical currents measured in our experiment. The current is normalized to the Ginzburg-Landau depairing current $I_{G L}(T)$.

function of the magnetic field (at $B<B_{a}^{*}$ ), we can conclude that the screening current in the loop does not influence the transition of our system to the normal state.

Therefore, it is justified to assume that the transition of the current leads to the normal state results simultaneously in a transition of the loop to the normal state when the reservoirs are superconducting. The critical current $I_{c}^{+}$is equal to the depairing current $I_{G L}(T) \simeq 1.94 \Delta_{0} S(1$ $\left.-T / T_{c}\right)^{3 / 2} / \xi_{0} \rho_{n} e$, where $\rho_{n}$ is the normal state resistivity and $S$ is the cross-section of the current lead. An estimation of $I_{G L}$ at $T=0.982 T_{c}$ for the parameters of the loop of Fig. 1 gives us a value which is only $30 \%$ larger than found in the experiment $\left(\rho_{n} \simeq 2.2 \times 10^{-7} \Omega \mathrm{m}\right)$. Obviously, the current $\left|I_{c}^{-}\right|$ cannot be larger than $I_{G L}$. The cause of the hysteresis in the current-voltage $(I-V)$ characteristics at low temperatures and low magnetic fields (at $B<B_{a}^{*}$ ) is not clear and needs further investigation.

The case when the banks are in the normal state is different. At the place where the current leads are attached to the banks, a normal metal-superconductor (N-S) boundary forms and the normal current flowing from the normal regions converts to superconducting current over a finite length. ${ }^{6}$ The finite voltage drop between the normal banks leads to a nonequilibrium quasiparticle distribution function $f(E, x)$ in the superconductor and it affects the superconducting properties of our system. ${ }^{7,8}$ To quantify this, we studied the superconducting loop with two attached superconducting leads having N-S boundaries at their ends (see inset in Fig. 4) using the Usadel equations ${ }^{9}$ for the spatial dependence of the normal $\alpha(E)=\cos \Theta$ and anomalous $\beta(E)=\sin \Theta$ quasiclassical Green functions coupled to the Boltzmann equations for the distribution function $f(E, x)=\left[1-f_{L}(E, x)\right.$ $\left.-f_{T}(E, x)\right] / 2,{ }^{10-12}$

$$
\frac{d^{2} \Theta}{d x^{2}}+\left[\left(2 i E-\frac{1}{l_{E}^{2}}\right)-(\nabla \phi)^{2} \cos \Theta\right] \sin \Theta+2|\Delta| \cos \Theta=0,
$$

$$
\begin{aligned}
& \nabla\left[\left(N_{1}^{2}-R_{2}^{2}\right) \nabla f_{L}\right]+2 N_{2} R_{2} \nabla \phi \nabla f_{T}-\frac{N_{1}}{l_{E}^{2}}\left(f_{L}-f_{L}^{0}\right)=0, \\
& \nabla\left[\left(N_{1}^{2}+N_{2}^{2}\right) \nabla f_{T}\right]+2 N_{2} R_{2} \nabla \phi \nabla f_{L}-\frac{N_{1}}{l_{E}^{2}}\left(f_{T}-\varphi \frac{\partial f_{L}^{0}}{\partial E}\right) \\
& \quad-2 N_{2}|\Delta| f_{T}=0,
\end{aligned}
$$

where $f_{L}(E)$ and $f_{T}(T)$ are so-called longitudinal and transverse parts of $f(E)$, respectively. ${ }^{10}$ In Eqs. (1a)-(1c), $\phi$ is the phase of the order parameter $\Delta=|\Delta| e^{i \phi}, \varphi$ is an electrostatic potential, $\quad N_{1}(E)+i R_{1}(E)=\cos \Theta(E), \quad N_{2}(E)+i R_{2}(E)$ $=\sin \Theta(E)$, and $f_{L}^{0}(E)=\tanh (E / 2 T)$ is the odd part of the equilibrium Fermi-Dirac distribution function of the quasiparticles. The dimensionless length $l_{E}=\sqrt{D \tau_{E}} / \xi_{0}=L_{E} / \xi_{0}$ $=\sqrt{\tau_{E} \Delta_{0} / \hbar}$ defines the range over which the nonequilibrium distribution of the quasiparticles can exist in the sample. Here, $D$ is a diffusion constant and $\Delta_{0}$ is a zero-temperature value of the order parameter $\Delta_{0} \simeq 1.76 k_{B} T_{c}$ (in weak coupling limit). The characteristic relaxation time due to inelastic electron-phonon collisions depends on the energy $\tau_{E}(E)$. For low temperature superconductors, it can be taken at the Fermi energy $E_{F}$ because deviations from equilibrium occur mainly for $|E| \lesssim \pm|\Delta| \ll E_{F}$ [the energy in Eqs. (1a)-(1c) is counted from the Fermi level]. In this energy interval, $\tau_{E}$ varies slowly for $T \sim T_{c}$ (see, for example, Figs. 1 and 2 in Ref. 13).

In Eqs. (1a)-(1c), the order parameter $\Delta$ and energy are scaled by the $\Delta_{0}$, and distance is in units of the zerotemperature coherence length $\xi_{0}=\sqrt{\hbar D / \Delta_{0}}$. The current is scaled in units of $j_{0}=\Delta_{0} / \xi_{0} \rho_{n} e$ and the electrostatic potential is in units of $\varphi_{0}=\Delta_{0} / e\left(\rho_{n}\right.$ is the normal state resistivity and $e$ is the electric charge).

The order parameter distribution is determined by the Ginzburg-Landau equation ${ }^{11}$

$$
a_{1} \frac{d^{2} \Delta}{d x^{2}}+\left(1-\frac{T}{T_{c}}-a_{2}|\Delta|^{2}+\Psi_{1}+i \Psi_{2}\right) \Delta=0,
$$

with $a_{1} \simeq 0.69, a_{2} \simeq 0.33$, and the additional terms $\Psi_{1}$ $=\int_{0}^{\infty} R_{2}\left(f_{L}-f_{L}^{0}\right) d E /|\Delta|$ and $\Psi_{2}=\int_{0}^{\infty} N_{2} f_{T} d E /|\Delta|$ (for details, see Ref. 8).

The current and electrostatic potential in the system can be found from the following equations:

$$
\begin{gathered}
j=2 a_{1}|\Delta|^{2} \nabla \phi+\int_{0}^{\infty}\left[\left(N_{1}^{2}+N_{2}^{2}\right) \nabla f_{T}\right. \\
\left.+2 N_{2} R_{2}\left(f_{L}-f_{L}^{0}\right) \nabla \phi\right] d E, \\
\varphi=\int_{0}^{\infty} N_{1} f_{T} d E .
\end{gathered}
$$

As boundary conditions for Eqs. (1) and (2), we used the following relations:

$$
\Theta(\text { ends })=\Delta(\text { ends })=0,
$$






FIG. 5. (Color online) Distribution of the order parameter (solid symbols) and the electrostatic potential (open symbols) along our model system (at $T=0.98 T_{c}$ ) for two voltages which roughly correspond to $I \simeq I_{c}^{+}$and $I \simeq I_{c}^{-}$. In both cases, the superconducting voltage leads near the loop will measure zero voltage although there is a voltage drop near the loop.

$$
\begin{aligned}
& f_{L}(\text { ends })=\frac{1}{2}\left(\tanh \left[\frac{E+V(\text { ends })}{1.14 T / T_{c}}\right]+\tanh \left[\frac{E-V(\text { ends })}{1.14 T / T_{c}}\right]\right), \\
& f_{T}(\text { ends })=\frac{1}{2}\left(\tanh \left[\frac{E+V(\text { ends })}{1.14 T / T_{c}}\right]-\tanh \left[\frac{E-V(\text { ends })}{1.14 T / T_{c}}\right]\right),
\end{aligned}
$$

which model a direct electrical contact of our system to the normal banks for an applied voltage $\pm V$ at the ends.

The coupled nonlinear differential equations (1a)-(1c) and (2) are solved numerically using the finite difference technique (for details of numerical procedure, see Ref. 8). For our numerical calculations, the following parameters were used: $L_{\text {lead }}=50 \xi_{0}, R=8 \xi_{0}$ (which are close to the parameters of our experimental sample with $\xi_{0} \simeq 117 \mathrm{~nm}$ ), and $l_{E}$ $=32$ (we used a twice smaller value than the typical one for aluminum, ${ }^{14} L_{E}=\sqrt{D \tau_{E}} \sim 7 \mu \mathrm{m}$; see arguments below). We used a one-dimensional model and neglected the nonzero width of the leads and of the loop, as they are much smaller than $\xi(T)$ in the temperature range of interest.

In Fig. 4, we show the calculated $I-V$ characteristics at different temperatures in the absence of an applied magnetic field. ${ }^{15}$ In contrast to Fig. 2, the theoretical curves were obtained in the voltage-driven regime and we plotted curves only at positive voltages (currents). Note that for negative voltages, we have $I(-V)=-I(V)$. It is important to mention here that in the experiment, superconducting leads were used to measure the voltage. Consequently, the electrochemical potential of the superconducting electrons is measured, which is different from the electrostatic potential. ${ }^{6}$ When the order parameter in the loop is nonzero, the experimentally measured potential difference is zero although there can be a finite voltage drop in the loop (see Fig. 5). In Fig. 4, all points on the $I-V$ characteristics meet this condition. Therefore, in the experiment with superconducting voltage leads, we measure zero voltage for $I_{c}^{-}<I<I_{c}^{+}$, and the arrows in

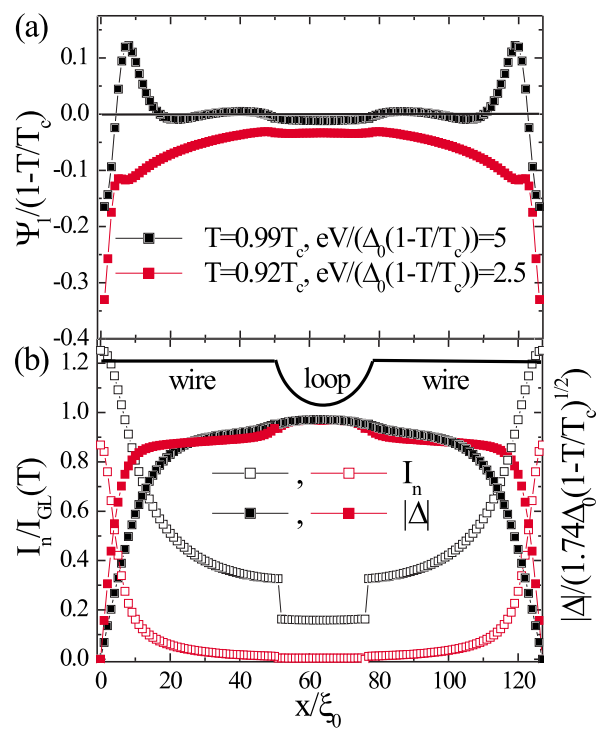

FIG. 6. (Color online) Distribution of the (a) potential $\Psi_{1}$, normal current, and (b) order parameter along our system for relatively low $\left(T=0.92 T_{c}\right)$ and high $\left(T=0.99 T_{c}\right)$ temperatures.

Fig. 4 indicate the points which are identified as the experimentally observable critical currents in the negative and positive directions. Only when the order parameter is equal to zero in the loop (at $I<I_{c}^{-}$and at $I>I_{c}^{+}$) do the superconducting voltage leads measure the electrostatic potential, which in the experiment is observed as a sharp jump at the critical currents.

We may distinguish two temperature ranges: the range of high temperatures, where current $I_{c}^{+}=\left|I_{c}^{-}\right|$, and the range of low temperatures, where $I_{c}^{+}>\left|I_{c}^{-}\right|$. The latter case should exhibit hysteresis in the current-driven regime. In different temperature ranges, different relaxation mechanisms for the nonequilibrium carriers will dominate, leading to differences in the $I-V$ characteristics.

In Fig. 6(a), we show the dependence of the potential $\Psi_{1}=\int_{0}^{\infty} R_{2}\left(f_{L}-f_{L}^{0}\right) d E /|\Delta|$, which describes the suppression of $|\Delta|$ by the nonequilibrium $f_{L}$ at different temperatures. Due to the presence of the coupling term $2 N_{2} R_{2} \nabla \phi \nabla f_{T}$ in Eq. (1b), a fast relaxation of $f_{L}$ occurs at the N-S boundaries ${ }^{8}$ on the scale of the coherence length. In Fig. 6(a), this corresponds to a fast decay of $\Psi_{1}$ at the ends of our system. At low temperature, the coherence length is short, and this decay will not be strong. As a result, the relaxation of $f_{L}$ mainly occurs due to the finite time $\tau_{E}$, and the characteristic decay length is $L_{E}$ [leading to a much longer decay of $\Psi_{1}$; see Fig. 6(a)]. Because the length of the leads $L_{\text {lead }}$ is comparable to $L_{E}$, the relaxation is not complete and the voltage induced potential $\Psi_{1}$ suppresses the order parameter both in the leads and in the loop. This, in turn, leads to a decrease of the critical current of our system below the depairing current. ${ }^{7}$ Experimentally, this is observed as an abrupt drop in $I_{c}^{+}$at $T=0.962 T_{c}$ when the N-S boundaries form at magnetic field $B_{a}^{*}$ (at this moment, the potential $\Psi_{1}$ appears abruptly in our system). The suppression of the order parameter leads to the $\mathrm{S}$-shape behavior of the $I-V$ characteristic (see Fig. 4) that could be found in experiment when one uses normal voltage leads. 


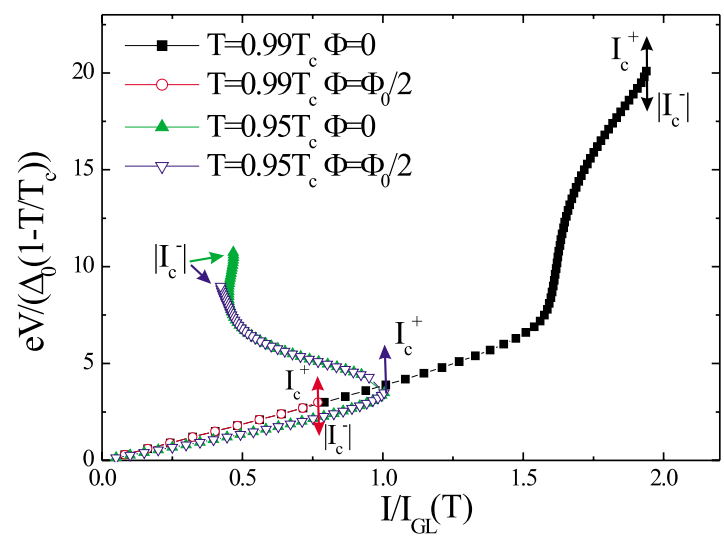

FIG. 7. (Color online) Current-voltage characteristics of our model system at zero and half magnetic flux quantum through the loop. At high temperature, both currents $I_{c}^{ \pm}$vary with magnetic field, while at low temperature, only the current $I_{c}^{-}$changes.

For high temperatures, due to the fast increase of the coherence length, the additional decay of $f_{L}(E)$ at the N-S boundary becomes essential [see Fig. 6(a)]. This additional mechanism effectively relaxes the nonequilibrium $f_{L}$, and the potential $\Psi_{1}$ is mainly nonzero only near the N-S boundaries (where it even may change the sign and, hence, enhance the superconducting properties). Therefore, the suppression of the order parameter in the loop due to deviations of $f_{L}$ from equilibrium is effectively weakened in the high temperature regime.

However, at high temperature, another effect becomes important, which is connected with the nonequilibrium distribution function. Because $f_{T}$ is nonzero in the superconductor, a normal current $I_{n} \sim \int_{0}^{\infty}\left(N_{1}^{2}+N_{2}^{2}\right) \nabla f_{T} d E$ can exist in the superconductor [see Fig. 6(b)]. Its penetration occurs on a length scale somewhere in between $\xi(T)$ and $L_{E}$ [if $L_{E}$ $>\xi(T)]$. The reason is that the part of the normal current that is converted to a superconducting one near N-S boundaries via Andreev reflection occurs over a length scale $\xi(T)$ (for quasiparticles with energies less than $|\Delta|$ ), and the other part of the normal current is converted via inelastic relaxation of the nonequilibrium quasiparticles having a length scale $L_{E}$ (for quasiparticles with energies larger than $|\Delta|$ ), see Eq. (1c).

Because $\xi(T)$ increases with temperature, the normal component of the current penetrates further in our system. Since the total current is equal to the sum of the superconducting $I_{s}$ and the normal $I_{n}$ current, a higher current has to be applied in order to satisfy the condition $I_{c}^{+}=I_{s} \simeq I_{G L}$ in our system. At low temperature, the normal current in the loop and leads is small [except for regions near the N-S boundaries; see Fig. 6(b)] and it has a small effect on the critical current. On the contrary, at high temperature, the normal current penetrates far into the superconductor and the critical current $I_{c}^{+}$of the N-S-N structure may exceed the depairing value $I_{G L}$. In the experiment, this has been observed as an abrupt increase of $I_{c}^{ \pm}$at $T=0.982 T_{c}$ when the N-S boundaries form at the ends of the current leads and the normal current appears abruptly in the loop.

The appearance of hysteresis and the oscillating behavior of $I_{c}^{-}\left(B_{a}\right)$ at low temperature $\left(I_{c}^{+}\right.$does not oscillate up to a

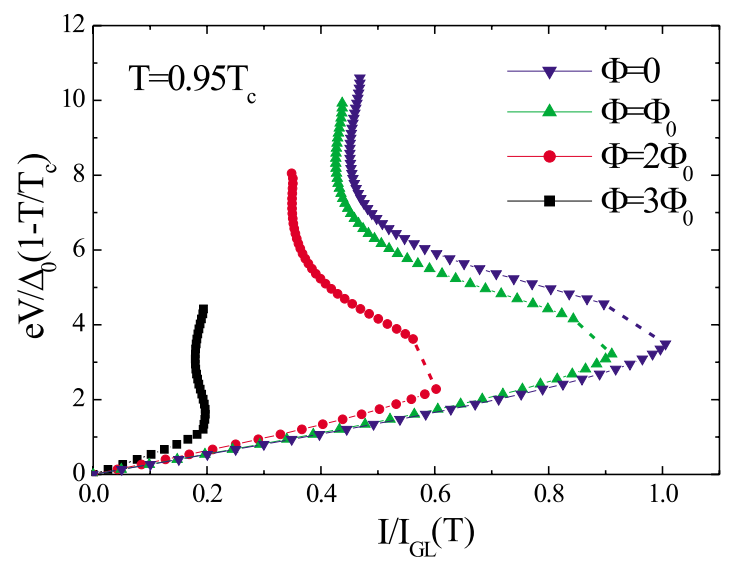

FIG. 8. (Color online) Current-voltage characteristics of our model system at different magnetic fields at $T=0.95 T_{c}$. There is a gradual decrease of the hysteresis in the current-driven regime with increasing magnetic field (compare with Fig. 4). Dashed lines mark the voltage interval where there is no stationary superconducting state in the current leads.

magnetic filed much larger than $B_{a}^{*}$ ) are also accounted for by our model. Indeed, with decreasing temperature, the difference in critical currents increases (see Fig. 4). At high voltages $\left(I \sim I_{c}^{-}\right)$, superconductivity survives only in the loop, but it is strongly suppressed by the voltage induced nonequilibrium quasiparticles (see Fig. 5). In this case, the effect of the screening currents becomes important and, as a result, the $I-V$ characteristics become dependent on the applied magnetic flux for currents close to $I_{c}^{-}$(see Fig. 7). However, for current $I=I_{c}^{+}$, the order parameter in the loop is practically not suppressed (see Fig. 5) and the effect of the screening currents is negligible (see Fig. 7). At high temperatures, the order parameter is strongly suppressed in the loop not only at $I \sim I_{c}^{-}$but at $I \sim I_{c}^{+}$too. It provides strong dependence of both currents $I_{c}^{ \pm}$on the applied magnetic field at high temperatures (see Fig. 7).

When the critical field of the leads and the loop is approached $\left(B_{c} \sim \Phi_{0} / \xi w\right)$, the order parameter becomes strongly suppressed in the system. The same occurs when temperature is increased. Therefore, we expect that, qualitatively, the $I-V$ characteristics depend on $B_{a}$ in the same way as on temperature, except for oscillations related with the screening current in the loop. In Fig. 8, we present our theoretical results which confirm that idea. ${ }^{16}$ With increasing magnetic field, hysteresis becomes weaker and, at some value, the $I-V$ characteristics become reversible in the current-driven regime, as was also found in the experiment [see Fig. 3(b)].

\section{DISCUSSION}

In our calculations, we used $l_{E}=32$ which is almost twice smaller than the typical value for aluminum, ${ }^{14} l_{E}^{\mathrm{Al}} \simeq 60$. If we use the latter value, we obtain qualitatively the same results as in Fig. 4, but at a temperature closer to $T_{c}$. This discrepancy between theory and experiment can be attributed to the presence of voltage leads in our system. Their width is the 
same as the linewidth of the loop and the current leads (see Fig. 1), and they serve as an effective drain for the relaxation of $f(E, x)$. In addition, the order parameter is larger in the voltage leads and, thus, enhances the order parameter in the loop. Furthermore, in our calculations, $L_{\text {lead }}=50 \xi_{0} \simeq 6 \mu \mathrm{m}$ has been used as the length of the current leads. The real length is larger because the current leads become wider at about $5 \mu \mathrm{m}$ from the loop and extend to $15 \mu \mathrm{m}$ before they reach the bulk superconductor. It is clear that in the wide lead (even being in the normal state), an additional relaxation of the nonequilibrium $f_{L}$ should occur. All the above factors will shift the temperature at which the critical current becomes smaller than the depairing current to lower values. Besides, they increase the region wherein superconductivity exists in the loop to larger voltages and also increase the current $\left|I_{c}^{-}\right|$, which is compared to the one found in our model system.

We should note that a similar enhancement of the critical current by applied magnetic field was recently observed in zinc nanowires. ${ }^{18}$ Similar to our work, this occurs when the magnetic field suppresses superconductivity in bulk superconductors. In contrast to our findings, the current enhancement was observed even at $T \sim 0.5 T_{c}$ and it becomes weaker when approaching $T_{c}$. We explain this difference by the presence of bulk superconductors ( $\mathrm{Sn}, \mathrm{In}$, and $\mathrm{Pb}$ ) having much shorter decay length $L_{E}$ than for zinc. The samples were made by mechanically squeezing high-purity superconducting $\mathrm{Pb}(\mathrm{Sn}, \mathrm{In})$ wires on both sides of the polycarbonate membrane, forming a $\mathrm{Pb} / \mathrm{ZNW} / \mathrm{Pb}$ structure. ${ }^{18}$ The method of preparation makes it possible to add another superconducting material at the end of the zinc nanowires that could provide an effective relaxation of $f(E, x)$ and suppress the effect of the applied voltage. The authors of Ref. 18 noticed that the observed current enhancement is weaker for a bulk material with a stronger electron-phonon interaction (thus, shorter $L_{E}$ ), in agreement with our assumption.

Our experimental results at low temperature ( $T$ $=0.962 T_{c}$ ) support the idea of Keizer et al. ${ }^{7}$ about the suppression of the superconductivity by an applied voltage. In Ref. 7, it was theoretically found that in a superconductor with N-S boundaries, the critical current (current at which the superconductivity vanishes in the sample) can be smaller than the depairing current. The origin of the effect is the same as in our case: the normal current creates the voltage drop between the normal banks and it strongly modifies the quasiparticle distribution function. In the experiment, we can suppress superconductivity in the banks and weakly affect the order parameter in the superconducting leads. As a result, we can directly compare the critical currents of our system with superconducting banks (when the critical current is equal to the depairing current) and with normal banks (when critical current is smaller than $I_{G L}$ at low temperatures).

\section{CONCLUSIONS}

In conclusion, we studied both experimentally and theoretically the transport properties of a superconducting loop connected by superconducting leads to bulk superconducting reservoirs. We found that when a sufficiently large magnetic field is applied, the superconducting reservoirs turn to the normal state and the response of our system drastically changes. The critical current of the system increases to values larger than $I_{c}$ at zero magnetic field (at temperatures close to $T_{c}$ ) and suppresses $I_{c}$ at lower temperatures. The last effect is explained by the strong influence of the nonequilibrium quasiparticle distribution function (induced by the voltage drop in the normal region) on the value of the order parameter. We expect that the observed phenomena should be most pronounced for loops with superconducting leads with a length smaller or comparable to the decay length of $f(E, x)$.

\section{ACKNOWLEDGMENTS}

This work was supported by the Flemish Science Foundation (FWO-Vl), the GOA/2004/02 Programme, the Belgian Science Policy (IUAP), and the ESF-AQDJJ program. D.Y.V. acknowledges support from INTAS Young Scientist Programme (04-83-3139) and Dynasty Foundation. *vodolazov@ipm.sci-nnov.ru

†resent address: Philips Semiconductors B.V., Kapeldreef 75, B-3001 Leuven, Belgium.

francois.peeters@ua.ac.be

${ }^{1}$ M. Lange, M. J. Van Bael, Y. Bruynseraede, and V. V. Moshchalkov, Phys. Rev. Lett. 90, 197006 (2003).

${ }^{2}$ M. Yu. Kharitonov and M. V. Feigel'man, JETP Lett. 82, 421 (2005).

${ }^{3}$ T. C. Wei, D. Pekker, A. Rogachev, A. Bezryadin, and P. M. Goldbart, Europhys. Lett. 75, 943 (2006).

${ }^{4}$ L. Balicas, J. S. Brooks, K. Storr, S. Uji, M. Tokumoto, H. Tanaka, H. Kobayashi, A. Kobayashi, V. Barzykin, and L. P. Gor'kov, Phys. Rev. Lett. 87, 067002 (2001).

${ }^{5}$ D. S. Golubović and V. V. Moshchalkov, Appl. Phys. Lett. 87, 142501 (2005).
${ }^{6}$ M. Tinkham, Introduction to Superconductivity (McGraw-Hill, New York, 1996), p. 421.

${ }^{7}$ R. S. Keizer, M. G. Flokstra, J. Aarts, and T. M. Klapwijk, Phys. Rev. Lett. 96, 147002 (2006).

${ }^{8}$ D. Y. Vodolazov and F. M. Peeters, arXiv:cond-mat/0611315 (unpublished).

${ }^{9}$ K. D. Usadel, Phys. Rev. Lett. 25, 507 (1970).

${ }^{10}$ A. Schmid and G. Schön, J. Low Temp. Phys. 20, 207 (1975).

${ }^{11}$ L. Kramer and R. J. Watts-Tobin, Phys. Rev. Lett. 40, 1041 (1978).

${ }^{12}$ R. J. Watts-Tobin, Y. Krähenbühl, and L. Kramer, J. Low Temp. Phys. 42, 459 (1981).

${ }^{13}$ S. B. Kaplan, C. C. Chi, D. N. Langenberg, J. J. Chang, S. Jafarey, and D. J. Scalapino, Phys. Rev. B 14, 4854 (1976); 15, 3567(E) (1977). 
${ }^{14}$ M. Stuivinga, J. E. Mooij, and T. M. Klapwijk, J. Low Temp. Phys. 46, 555 (1982).

${ }^{15}$ We can neglect here the influence of low magnetic field on the order parameter in the current leads because only a small decay of the critical current is found in experiment (see Fig. 3) when the magnetic field is about $B_{a}^{*}$.

${ }^{16}$ In our one-dimensional equations, the effect of magnetic field was taken into account by adding in the left-hand side of Eq. (3) the additional term $-\Delta\left(H / H_{c}\right)^{2}$, which allows us to accurately take into account the gradual suppression of the order parameter by the applied magnetic field in narrow superconductors (Ref. 17). ${ }^{17}$ D. Y. Vodolazov, Phys. Rev. B 75, 184517 (2007).

${ }^{18}$ M. Tian, N. Kumar, J. G. Wang, S. Y. Xu, and M. H. W. Chan, Phys. Rev. B 74, 014515 (2006). 